\title{
Efficacy of imiquimod $5 \%$ cream as first-line management in cutaneous leishmaniasis caused by Leishmania mexicana
}

\author{
Gabriela Fuentes-Nava ${ }^{[1]}$, Andrés Tirado-Sánchez ${ }^{[1],[2], ~ E d i t h ~ A . ~ F e r n a ́ n d e z-F i g u e r o a ~}{ }^{[3],[4]}$, \\ Sokani Sánchez-Montes ${ }^{[4],[5], ~ I n g e b o r g ~ B e c k e r ~}{ }^{[4]}$ and Alexandro Bonifaz ${ }^{[1]}$
}

\author{
[1]. Hospital General de México, Servicio de Dermatología, México. \\ [2]. Instituto Mexicano del Seguro Social, Hospital General de Zona 29, Departamento de Medicina Interna, México. \\ [3]. Instituto Nacional de Medicina Genómica, Laboratorio de Genómica Computacional, México. \\ [4]. Universidad Nacional Autónoma de México, Facultad de Medicina, Centro de Medicina Tropical, Unidad de Medicina Experimental, México. \\ [5]. Universidad Veracruzana, Facultad de Ciencias Biológicas y Agropecuarias, México.
}

\begin{abstract}
Cutaneous leishmaniasis (CL) involves several differential diagnoses as it lacks a gold standard diagnostic test. Its diagnosis is easier in endemic regions; however, many cases come from travelers to endemic areas. A 22-year-old patient, who had recently visited Oaxaca, Mexico, developed two asymptomatic ulcers weeks later on the left auricle and the nose. Leishmania mexicana was identified using polymerase chain reaction. The patient was treated with imiquimod 5\% cream three times/week, providing favorable results after 12 weeks, without relapse 2 months after therapy. To our knowledge, this is the first case of CL due to L. mexicana effectively treated with imiquimod.
\end{abstract}

Keywords: Leishmaniasis. Imiquimod. Treatment.

\section{INTRODUCTION}

Leishmaniasis is an infectious disease caused by different intracellular protozoal species related to the genus Leishmania (L.) and transmitted to humans from a female hematophagous of the Diptera order from the genus Lutzomyia (in America) or Phlebotomus (in Europe, Asia, and Africa) $)^{1,2}$, which regurgitates promastigotes. Leishmaniasis can be classified into three primary clinical forms: cutaneous (localized or diffused), mucocutaneous, and visceral leishmaniasis (or kala-azar) ${ }^{1,3}$. In Mexico, cutaneous leishmaniasis is endemic to Campeche, Chiapas, Coahuila, Jalisco, Michoacán, Nayarit, Nuevo León, Oaxaca, Tamaulipas, Quintana Roo, Tabasco, Veracruz, Yucatán, and Sinaloa ${ }^{4}$. Several treatments have been tested for cutaneous leishmaniasis with different outcomes. Imidazoquinolines, including imiquimod, are low-molecular-weight substances that can regulate immune responses through interactions with toll-like receptors on cells.

\footnotetext{
Corresponding autor: Dr. Andrés Tirado-Sánchez.

e-mail: atsdermahgm@gmail.com

(i) https://orcid.org/0000-0001-9306-1619

Received 1 June 2020

Accepted 2 September 2020
}

Imiquimod acts via immunomodulatory activity on several immune cells (Langerhans cells), regulating the release of proinflammatory cytokines (IFN- $\gamma$, TNF- $\alpha$, IL-1 $\beta$, IL-1 $\alpha$, IL-6, IL-1) receptor antagonists, granulocyte-macrophage colony-stimulating factor and granulocyte colony-stimulating factor and also regulates nitric oxide-mediated phagocytosis ${ }^{5}$. Although other topical to intralesional alternatives for the management of cutaneous leishmaniasis, such as pentamidine isethionate or pentavalent antimonial have been tested with good results, many of these are not available in Mexico; therefore, the search for new treatment options is required. We report the first case of localized cutaneous leishmaniasis caused by L. mexicana successfully treated with imiquimod 5\% cream as the first-line therapy.

\section{CASE REPORT}

A previously healthy 22-year-old man presented to our dermatology department with an 8-week skin lesion that developed 20 weeks after traveling to the Chacahua Lagoons in the state of Oaxaca. We observed a $13 \times 18 \mathrm{~mm}$ ulcerated nodule in the dorsum of the nose and left ear (Figure 1a and Figure 2a, respectively). The patient was previously treated with antibiotics without success. An ear biopsy (6 mm) was performed followed by DNA extraction using the High Pure PCR Template Preparation Kit (Roche, Cat.11796828001). Human glyceraldehyde-3-phosphate 


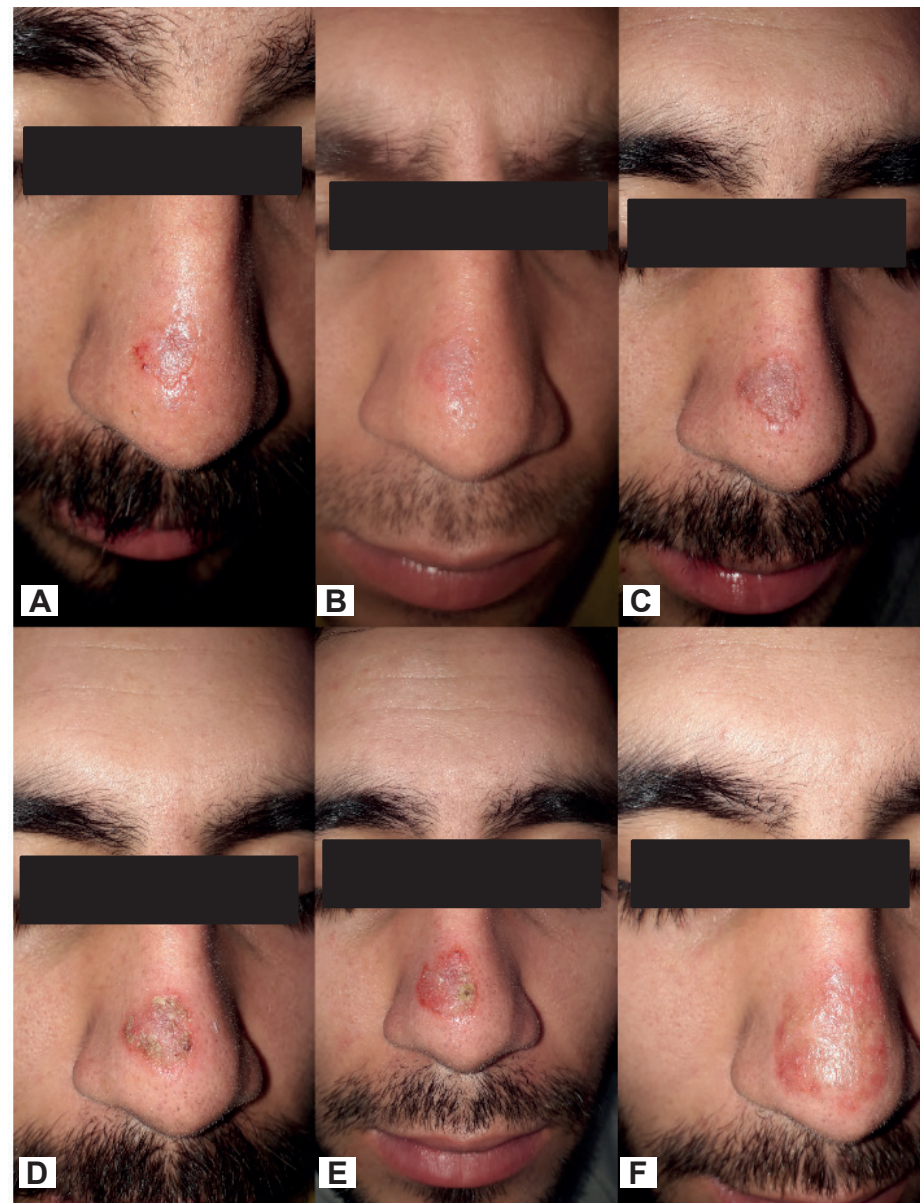

FIGURE 1: (A) a $13 \times 18 \mathrm{~mm}$ ulcerated nodule in the dorsum of the nose; (B) Two weeks of treatment with imiquimod $5 \%$ cream; (C) Four weeks of treatment; (D) Eight weeks of treatment; (E) Twelve weeks of treatment; (F) Two months after termination of treatment.

dehydrogenase was amplified by quantitative PCR (qPCR), as reported previously ${ }^{6}$, to evaluate the DNA integrity of the clinical sample. Further, a qPCR assay (qPCR-ama) targeting kDNA minicircles was performed as previously described ${ }^{7}$. As positive controls, DNA isolated from L. amazonensis MHOM/ BR/00/LTB0016, L. amazonensis IFLA/BR/67/PH8, L. mexicana MHOM/MX/2011/Lacandona, and L. mexicana isolate 14 were

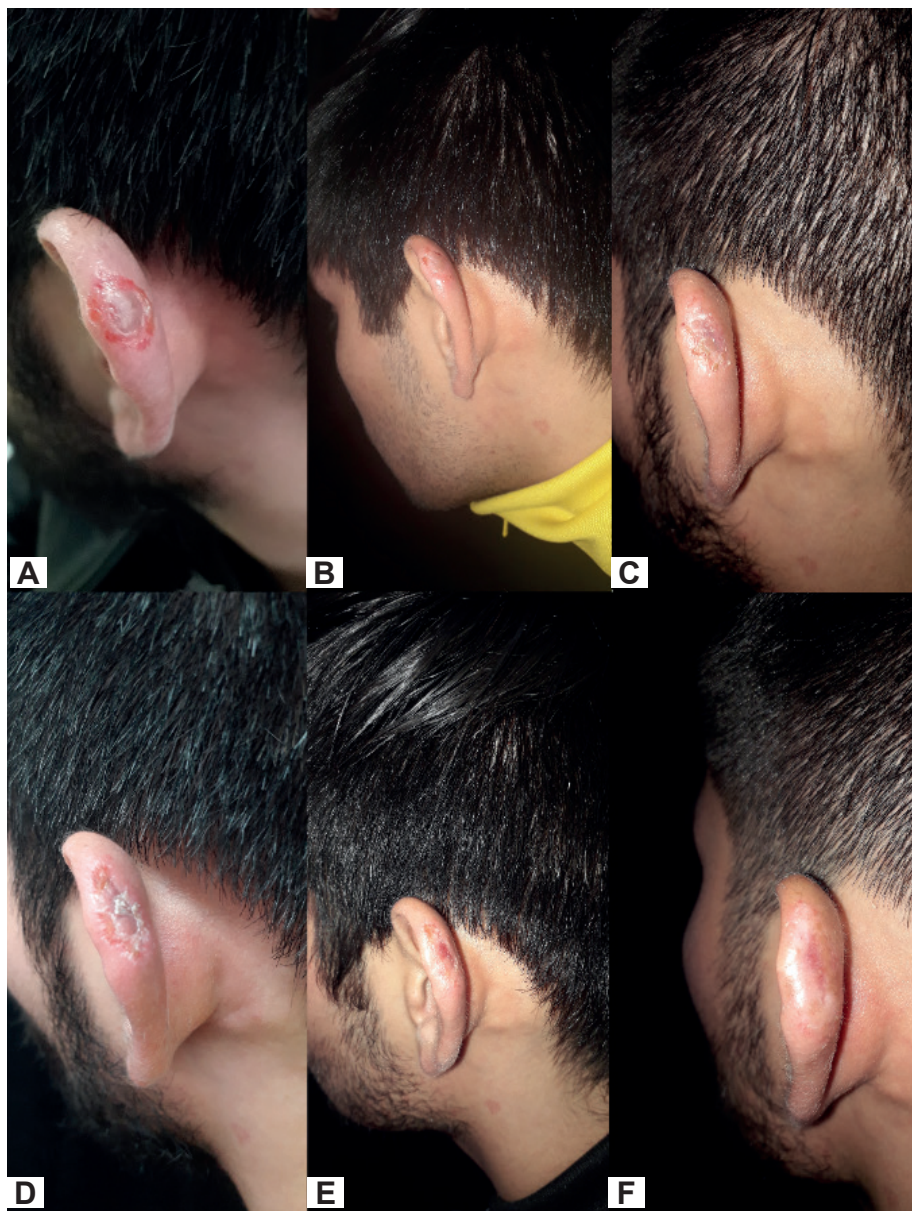

FIGURE 2: (A) an ulcerated nodule in the left atrial pavilion; (B) Two weeks of treatment with imiquimod 5\% cream; (C) Four weeks of treatment; (D) Eight weeks of treatment; (E) Twelve weeks of treatment; (F) Two months after termination of treatment.

used as templates. Non-template reactions were used as negative controls. The sample tested positive for L. mexicana (Figure 3). The treatment options were formulated based on the size of the lesion; however, the patient refused systemic treatment; therefore, we decided to prescribe $5 \%$ imiquimod cream, achieving a favorable response at 12 weeks (Figure 1b-e; Figure 2b-e) and no relapse at the 2-month follow-up (Figure 1f, Figure 2f).

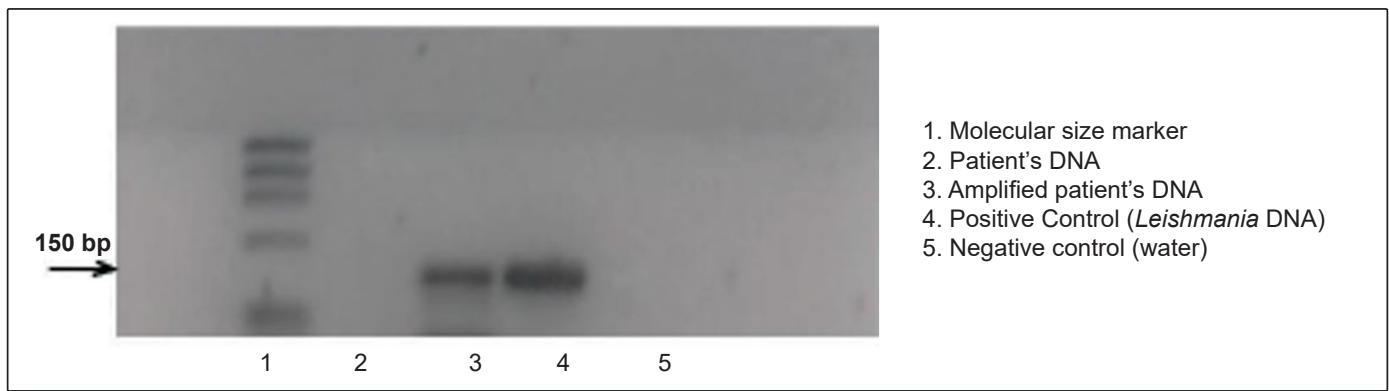

FIGURE 3: DNA extraction was done using the High Pure PCR Template Preparation Kit (Roche, Cat.11796828001). The DNA integrity of the clinical sample was evaluated with human glyceraldehyde-3-phosphate dehydrogenase (GAPDH) and it was amplified by qPCR. As positive controls, DNA isolated from Leishmania amazonensis MHOM/BR/00/LTB0016, L. amazonensis IFLA/BR/67/PH8, L. mexicana MHOM/MX/2011/Lacandona, and L. mexicana isolate 14 were used as templates. The sample was positive for L. mexicana. Labels: 1.Molecular size marker; 2.Patient's DNA; 3.Amplified patient's DNA; 4.Positive control (Leishmania DNA); and, 5.Negative control (water). 


\section{DISCUSSION}

Leishmaniasis is a parasitic infection with an uncertain frequency. Localized cutaneous leishmaniasis initially develops as a pruritic papule, progressing to an ulcer in exposed areas such as the ears, face, or arms. The definitive diagnosis is the identification of amastigotes inside the phagocytic cells isolated from the lesion by a stained imprint that facilitates microscopic identification or molecular tests (PCR).

Our results are consistent with those of previous reports. Córdoba-Uscanga et al. ${ }^{4}$, showed that L. mexicana is the most common causative agent of cutaneous leishmaniasis in Mexico.

Topical imiquimod, an immunomodulator and antineoplastic agent, has been used in the management of cutaneous leishmaniasis in two main contexts, that is, in combination with other treatments such as antimonials and cases resistant to conventional treatments, due to the leishmanicidal activity demonstrated by it in tissue culture and experimental studies ${ }^{8}$.

The management of cutaneous leishmaniasis is a therapeutic challenge due to the toxicity of the drugs and drug resistance; therefore, the treatment must be individualized according to disease severity, the etiological agent, and the host.

In cutaneous leishmaniasis, imiquimod stimulates interferon- $\gamma$ secretion by CD4 Thelper- 1 lymphocytes by activating macrophages to destroy amastigotes ${ }^{9}$. This mechanism of action could reduce resistance and treat areas with surrounding subclinical infection. In our patient, a moderate inflammatory reaction, which extended beyond the initial lesion, was observed at 4 weeks of use and was solved after 6 weeks without additional treatment or termination of the imiquimod treatment.

Some in vivo and in vitro ${ }^{10}$ reports have shown the effect of topically applied imiquimod on cutaneous leishmaniasis ${ }^{10-12}$. However, a recent systematic review established that imiquimod has no significant therapeutic effect when combined with antimonials ${ }^{13}$. Our patient achieved complete recovery after topical imiquimod treatment without previous treatments, and no relapse was observed after a 2-month follow-up. Moreover, spontaneous healing of cutaneous leishmaniasis may also occur, and this factor cannot be excluded in the present case; however, the treatment reduced the growth of the lesions, limiting complications, mainly scarring, and sped up the healing process.

To our knowledge, this is the first case of cutaneous leishmaniasis due to $L$. mexicana successfully treated with $5 \%$ imiquimod as a first-line therapy.

\section{AUTHORS' CONTRIBUTIONS}

GFN contributed with patient clinical care, data collection, literature search and writing; ATS contributed with data collection, literature search and writing; EFF contributed with data collection and laboratory test; SSM contributed with laboratorial test and data interpretation; IB contributed with data interpretation and writing; and $\mathrm{AB}$ contributed with patient clinical care, data collection, data interpretation and writing.

\section{CONFLICT OF INTEREST}

The authors declare that there is no conflict of interest.

\section{REFERENCES}

1. Salotra P, Singh R. Challenges in the diagnosis of post kala-azar dermal leishmaniasis. Indian J Med Res. 2005;123(3):295-310.

2. Hernández-Ruiz J, Becker I. Linfocitos T citotóxicos CD8+ en la leishmaniasis cutánea. Salud Publica Mex. 2006;48:430-9.

3. Reithinger R, Dujardin JC, Louzir H, Pirmez C, Alexander B, Brooker S. Cutaneous leishmaniasis. Lancet Infect Dis. 2007;7(9):581-96. doi:10.1016/S1473-3099(07)70209-8.

4. Córdova-Uscanga C, Albertos-Alpuche NE, Andrade-Naváez FJ, Canto-Lara SB. Leishmaniasis: estudio epidemiológico preliminar en una localidad de la zona endémica del estado de Tabasco. Salud Publica Mex. 1993;35(4):345-50.

5. Khalili G, Dobakhti F, Mahmoudzadeh-Niknam H, Khaze V, Partovi F. Immunotherapy with Imiquimod increases the efficacy of Glucantime therapy of Leishmania major infection. Iran J Immunol. 2011;8(1): 45-51.

6. Ceccarelli M, Galluzzi L, Diotallevi A, Andreoni F, Fowler H, Petersen $\mathrm{C}$, et al. The use of kDNA minicircle subclass relative abundance to differentiate between Leishmania (L.) infantum and Leishmania (L.) amazonensis. Parasit Vectors. 2017;10(1):239. doi: 10.1186/s13071-0172181-x.

7. Galluzzi L, De Santi M, Crinelli R, De Marco C, Zaffaroni N, Duranti A, et al. Induction of endoplasmic reticulum stress response by the indole3 -carbinol cyclic tetrameric derivative CTet in human breast cancer cell lines. PLoS One. 2012;7(8):e43249. doi: 10.1371/journal.pone.0043249.

8. Buates S, Matlashewski G. Treatment of experimental leishmaniasis with the immunomodulators imiquimod and S-28463: efficacy and mode of action. J Infect Dis. 1999;179(6):1485-94.

9. Berbert TRN, de Mello TFP, Wolf Nassif P, Mota CA, Silveira AV, Duarte GC, et al. Pentavalent Antimonials Combined with Other Therapeutic Alternatives for the Treatment of Cutaneous and Mucocutaneous Leishmaniasis: A Systematic Review. Dermatol Res Pract. 2018;2018:9014726.

10. Seeberger J, Daoud S, Pammer J. Transient effect of topical treatment of cutaneous leishmaniasis with imiquimod. Int $\mathrm{J}$ Dermatol. 2003;42(7):576-9.

11. Hervás JA, Martín-Santiago A, Hervás D, Rojo E, Mena A, Rocamora $\mathrm{V}$, et al. Old world Leishmania infantum cutaneous leishmaniasis unresponsive to liposomal amphotericin B treated with topical imiquimod. Pediatr Infect Dis J. 2012;31(1):97-100. doi: 10.1097/ INF.0b013e31822dfbf7.

12. El Hajj R, Bou Youness H, Lachaud L, Bastien P, Masquefa C, Bonnet PA, et al. EAPB0503: An Imiquimod analog with potent in vitro activity against cutaneous leishmaniasis caused by Leishmania major and Leishmania tropica. PLoS Negl Trop Dis. 2018;12:e006854. doi: 10.1371/journal.pntd.0006854.

13. Husein-ElAhmed H, Gieler U, Steinhoff M. Evidence supporting the enhanced efficacy of pentavalent antimonials with adjuvant therapy for cutaneous leishmaniasis: a systematic review and meta-analysis. J Eur Acad Dermatol Venereol. 2020. doi: 10.1111/jdv.16333. 